Bull. Mater. Sci., Vol. 22, No. 3, May 1999, pp. 141-151. (C) Indian Academy of Sciences.

\title{
Porous materials: A case study of supramolecular organization in materials design
}

\author{
C N R RAO \\ Chemistry and Physics of Materials Unit and CSIR Centre of Excellence in Chemistry, Jawaharlal Nehru Centre \\ for Advanced Scientific Research, Jakkur P.O., Bangalore 560064, India
}

\begin{abstract}
Supramolecular synthesis is being increasingly employed in materials design. In this article, the design of porous solids is discussed as a case study. Examples from recent work in the areas of open-framework inorganic materials, mesoporous solids and organic porous solids are presented to illustrate this important aspect of materials chemistry.
\end{abstract}

Keywords. Porous materials; supramolecular organization; materials design.

\section{Introduction}

The last decade has seen intense research activity in the chemistry of materials. Chemists have been able to make a variety of materials based on their understanding of structure-property relations. In particular, new synthetic methods have been developed to prepare novel materials, particularly those which are metastable (Rao 1999). An important addition to the synthetic arsenal of chemists is the use of supramolecular chemistry (Atwood et al 1996). In supramolecular organization, weak bonds between molecules arising from hydrogen bonding, hydrophobic, van der Waals or electrostatic interactions are made use of, instead of the conventional covalent bonds. Supramolecular organization itself is not new to materials science. A variety of biomaterials in Nature clearly result from supramolecular organization. However, laboratory synthesis based on supramolecular design has gained importance only during this decade. Of the many examples of supramolecular synthesis, that of zeolitic and mesoporous solids constitutes an excellent case study. In this article, we shall examine a few illustrative examples of supramolecular design of materials based on the investigations carried out in this laboratory. We restrict our discussion to a few porous solids which include open-framework inorganic materials, mesoporous solids and organic porous solids.

\section{Open-framework inorganic materials}

The area of open-framework inorganic materials is a vibrant area of research today because of the potential of these materials in sorption, catalysis and separation processes (Thomas 1994; Bowes and Ozin 1996). These materials have been the result of imaginative efforts of chemists to simulate zeolites and related aluminosilicates, and aluminophosphates, with porous properties. All these materials are synthesized by using structure-directing agents, generally organic amines.

We shall first examine two open-framework tin(II) phosphates, I and II, obtained by hydrothermal synthesis by employing DABCO (1,4-diazobicyclo[2,2,2]octane) and DEED $\left(N, N^{\prime}\right.$-diethylethylenediamine) as the structuredirecting agents and characterized by single crystal X-ray diffraction studies (Ayyappan et al 1998a). The structure of $\mathrm{I},\left[\mathrm{C}_{6} \mathrm{~N}_{2} \mathrm{H}_{14}\right]^{2+} 2\left[\mathrm{SnPO}_{4}\right]^{-} \cdot \mathrm{H}_{2} \mathrm{O}$, is made up of layers constructed by the networking of $\mathrm{SnO}_{3}$ and $\mathrm{PO}_{4}$ units. The connectivity between these units creates 4 - and 8 -membered rings, forming a layer along the $b c$ plane (figure 1). The individual layers are held together by the protonated DABCO molecules which occupy spaces in between the layers along with a water molecule. This is clearly a simple structure compared to the other known $\mathrm{Sn}(\mathrm{II})$ phosphates. The tin phosphate, II, $\left[\mathrm{C}_{6} \mathrm{~N}_{2} \mathrm{H}_{18}\right]^{2+} 2\left[\mathrm{SnPO}_{4}\right]^{-}$formed by DEED, however, is even simpler in that its structure involves edge-sharing of 4-membered $\mathrm{Sn}_{2} \mathrm{P}_{2} \mathrm{O}_{4}$ rings, forming one-dimensional chains in the form of ladders (figure 2). The amine molecule is situated between the ladders. Indeed, II can be considered to be an inorganic ladderane, not unlike the organic counterpart. In both I and II, there are strong hydrogen bond interactions between the anionic framework (layer in the case of $\mathbf{I}$, and chain in the case of II) and the protonated amine molecules.

The two structures formed by the $\mathrm{Sn}_{2} \mathrm{P}_{2} \mathrm{O}_{4}$ tecton described above are the simplest open-framework tin phosphate structures known so far. The layered structure of I can be made from the chain structure of II by the scheme shown in figure 3 (compare this with figure 1). In the proposed transformation, every second alternate 4-membered ring of II is broken (Sn-O-P bond, forming 
a new $\mathrm{P}=\mathrm{O}$ ), accompanied by a simple rotation of the existing $\mathrm{P}=\mathrm{O}$ group about a $\mathrm{P}-\mathrm{O}$ single bond giving rise to a $\mathrm{Sn}-\mathrm{O}-\mathrm{P}$ bond and new 4- and 8-membered rings. The broken line in figure 3 represents the new Sn-O-P bond formed. Such a formulation is consistent with the doubling of the $b$ axis in I compared to II. In general, $n$-edge-sharing 4-membered rings can give rise to a ring with $4 n-2(n-1)$ atoms. Or, if we add $n$ 4-membered rings to a $m$-membered ring, we get a ring with $m+2 n$ atoms ( $m$ and $n$ represent $\mathrm{T}$ atoms; $\mathrm{T}=\mathrm{Si}, \mathrm{P}$ in aluminosilicate zeolites and $\mathrm{Sn}, \mathrm{P}$ in the present case). We thus see how 6-, 8-, 10- and 12-membered rings are formed by 4-membered rings.

We shall now look at an interesting zinc phosphate, III, $\left[\mathrm{NH}_{3}\left(\mathrm{CH}_{2}\right)_{2} \mathrm{NH}\left(\mathrm{CH}_{2}\right)_{2} \mathrm{NH}_{3}\right]^{2+} 2\left[\mathrm{Zn}_{2} \mathrm{PO}_{4}\left(\mathrm{HPO}_{4}\right)\right]^{-}$which has interesting structural features including threecoordinated oxygens and infinite $\mathrm{Zn}-\mathrm{O}-\mathrm{Zn}$ chains (Neeraj et al 1999a). This framework structure results from the sharing of vertices by $\mathrm{ZnO}_{4}$ and $\mathrm{PO}_{4}$ tetrahedra. The connectivity between these units forms layers, which are anionic. The structure-directing agent, diethylenetriamine (DETA), is doubly protonated and occupies spaces between the layers. Thus, the entire structure can be considered to be made up of alternating anionic (inorganic) and cationic (organic) layers. The connectivity between the $\mathrm{ZnO}_{4}$ and $\mathrm{PO}_{4}$ units is such that it produces three distinct types of chains, labelled A-C. The A type chain consists of alternate stacks of three- and four-membered rings while the $B$ type chain is made up of only four-membered rings. The $\mathrm{C}$ type chain is made up of only three-membered rings. The chains are

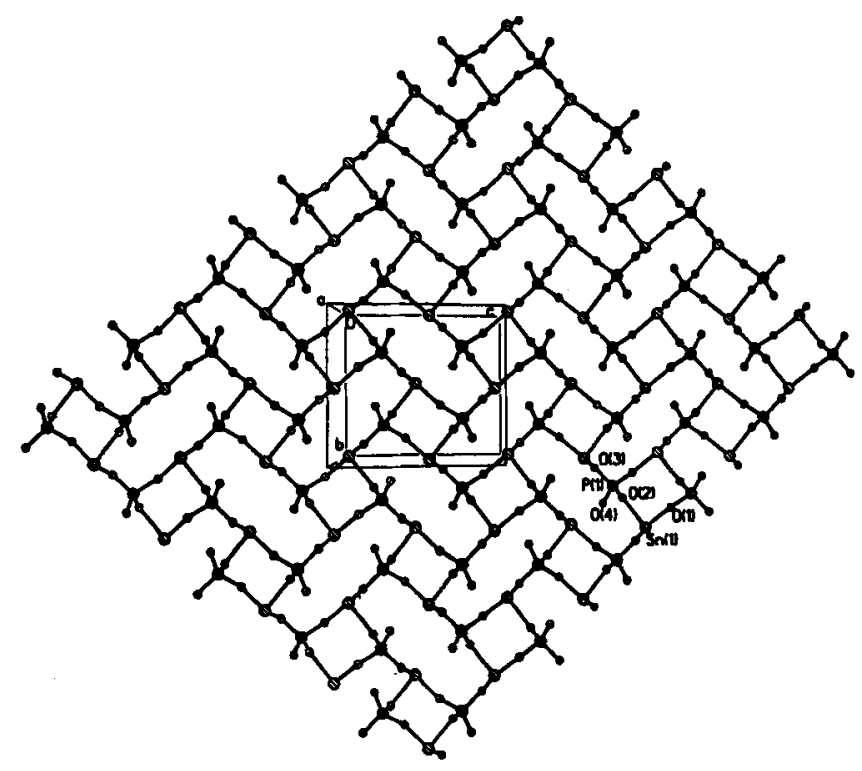

Figure 1. Structure of $\mathrm{I},\left[\mathrm{C}_{6} \mathrm{~N}_{2} \mathrm{H}_{14}\right]^{2+} 2\left[\mathrm{SnPO}_{4}\right]^{-} \cdot \mathrm{H}_{2} \mathrm{O}$ showing 4- and 8-membered rings and the connectivity within the layer (from Ayyappan et al 1998a). connected to one another forming the layer as shown in figure 4. To our knowledge, this zinc phosphate is the first example of an open-framework material where different types of chain arrangements are connected to each other forming a layer with ladder-like steps. Isolated and short chain three-membered rings are common in many of the open-framework zinc phosphates, but this is the first instance of a continuous three-membered ladder (chain) being present in an open-framework material. The presence of exclusive three-membered chains or ladders creates some strain in the layer, which is otherwise nearly planar and causes the formation of a step and a ladder-like feature in the material. Furthermore, we find an ABAC repeating unit along the $b c$ plane giving rise to the repeat ring sequence of 3343344433 along the $c$ axis (figure 4). When it is recalled that the three-membered ring is the smallest possible ring size and that two three-membered rings can make a four-member ring, the present structure can, in principle, evolve into a layer structure with four-membered rings, rarely seen in open-framework materials.

The framework of the zinc phosphate, III is also characterized by the presence of infinite of $\mathrm{Zn}-\mathrm{O}-\mathrm{Zn}$ chains. The $\mathrm{Zn}-\mathrm{O}-\mathrm{Zn}$ linkage is accompanied by the trigonal coordination of the bridging oxygen atoms, the third coordination being always to a $P$ atom. Such a trigonal coordination of the oxygen atom in the $\mathrm{Zn}-\mathrm{O}-\mathrm{Zn}$ bridge is an electrostatic valence requirement of the bridging oxygen atoms. Twenty-five percent of the oxygens in the asymmetric unit are three-coordinated (two of the eight framework oxygen atoms). Thus, it can be concluded that the presence of three-coordinated oxygen bridges does not give rise to more dense frameworks. The trigonal and tetrahedral coordination of the oxygen atoms observed in some of the structures reported in the literature suggests that these bridges occur when divalent tetrahedral atoms are involved. It is therefore to be expected that the presence of such features in the zinc phosphate system would lead to novel open-framework topologies which have no structural counterparts in aluminosilicates or aluminophosphates. The infinite one-dimensional $\mathrm{Zn}-\mathrm{O}-\mathrm{Zn}$ chain is formed only by the $\mathrm{Zn}$ (1) atoms (forming the three-membered ladders), but isolated short chain $\mathrm{Zn}-\mathrm{O}-\mathrm{Zn}$ linkages involving $\mathrm{Zn}(1)$ and other $\mathrm{Zn}$ atoms are also present.

An interesting aspect of this layered zinc phosphate is the connectivity between the $\mathrm{Zn}$ atoms within each layer. The $\mathrm{Zn}$ subnetwork resembles the fish backbone, a feature seen for the first time in this study. The $\mathrm{Zn}(1)$ atoms form the vertebrae onto which $\mathrm{Zn}(2)$ atoms are grafted, completing the fish backbone arrangement. This type of connectivity may be attributed to the presence of the three-membered chains and the three-coordinated oxygen atoms. 
We have recently synthesized a chiral zinc phosphate, IV, under hydrothermal conditions by employing the same achiral structure-directing amine as in the previous zinc phosphate (Neeraj et al 1999b). This phosphate has the composition, $\left[\mathrm{NH}_{3}\left(\mathrm{CH}_{2}\right) \mathrm{NH}_{2}\left(\mathrm{CH}_{2}\right)_{2} \mathrm{NH}_{3}\right]^{3+}\left[\mathrm{Zn}_{4}\left(\mathrm{PO}_{4}\right)_{3}\right.$ $\left(\mathrm{HPO}_{4}\right)^{3-}$. The most interesting aspect of this $\mathrm{Zn}$ phosphate is that it crystallizes in a polar space group $P Z_{1}$. The entire framework can be considered to be built from the networking of three-, four-, six- and eight-membered rings. The three- and four-membered rings are connected together, edge-wise, forming one-dimensional helical columns along the $b$ axis as shown in figure 5 which shows how these columns are interconnected via the $\mathrm{HPO}_{4}$ group forming an eight-membered channel along the $a$ axis. This eight-membered channel is connected to another eight-membered channel along the $b$ axis, forming a helical interconnected one-dimensional channel system where the amine and water molecules get situated. Thus, the material possesses an interpenetrating eight-membered channel system. There is strong hydrogen bonded interaction between the framework and the structure- directing amine providing structural stability. The framework density of this material is comparable to that of AIPO molecular sieves. It should be mentioned here that an interesting chiral open-framework of a $\mathrm{Sn}$ (II) phosphate has been made recently using guanidine as the structure-directing agent (Ayyappan et al 1998b).

An interesting 3-dimensional open-framework Sn(II) phosphate, $\mathbf{V}$, that we have made recently exhibits reversible dehydration and ion exchange properties (Natarajan et al 1998). This material, $\mathbf{V}$, has the composition $\left[\mathrm{Sn}_{4} \mathrm{P}_{3} \mathrm{O}_{12}\right]^{-} \quad\left[\left(\mathrm{NH}_{3} \mathrm{CH}_{2} \mathrm{CH}_{2} \mathrm{CH}\left(\mathrm{NH}_{3}\right) \mathrm{CH}_{2} \mathrm{CH}_{3}\right)^{2+}\right]_{0.5} \cdot 2 \mathrm{H}_{2} \mathrm{O}$. The entire framework structure can be considered to be made from the networking of four-, six-, eight- and twelve-membered rings formed by the $T$ atoms $(T=S n$, $P)$. The four-, six- and twelve-membered rings are connected to each other in such a way that they form a uniform 8-ring channels along the $a$ and $b$ axes (figures $6 \mathrm{a}$ and b). The widths of these channels are $7.0 \times 6.1 \AA$ along the $a$ axis and $8.3 \times 8.8 \AA$ along the $b$ axis (longest and shortest atom-atom contact distances, not including van der Waals radii). The widths of the channels suggest
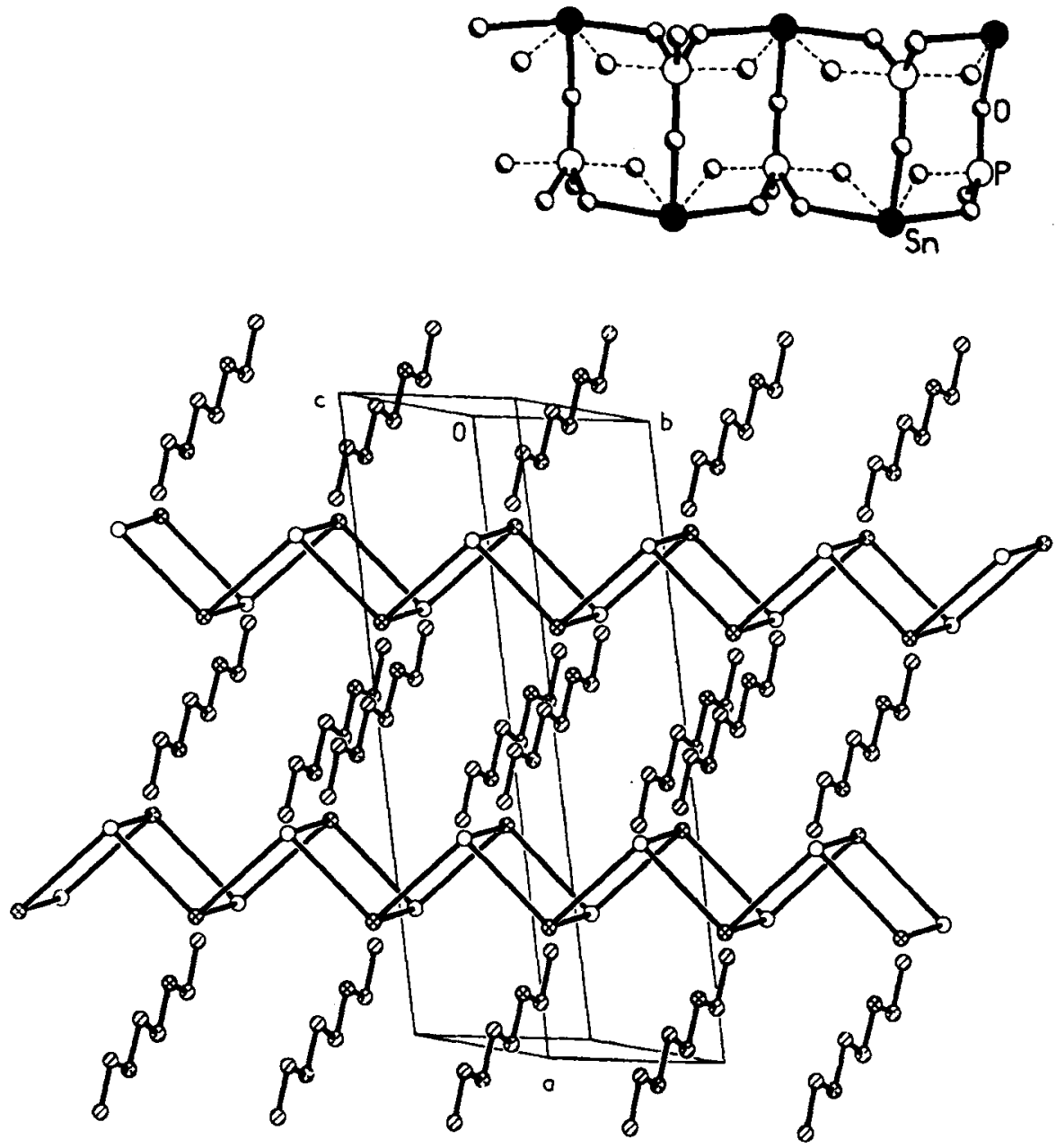

Figure 2. Structure of $\mathrm{II},\left[\mathrm{C}_{6} \mathrm{~N}_{2} \mathrm{H}_{18}\right]^{2+} 2\left[\mathrm{SnPO}_{4}\right]^{-}$showing the ladders and the amine (from Ayyappan et al 1998a). 
that the eight-membered channels along the $b$ axis are more regular (figure $6 \mathrm{~b}$ ) and the amine molecules sit in the middle of these channels. When viewed along the $a$ axis, the two water molecules occupy the distorted

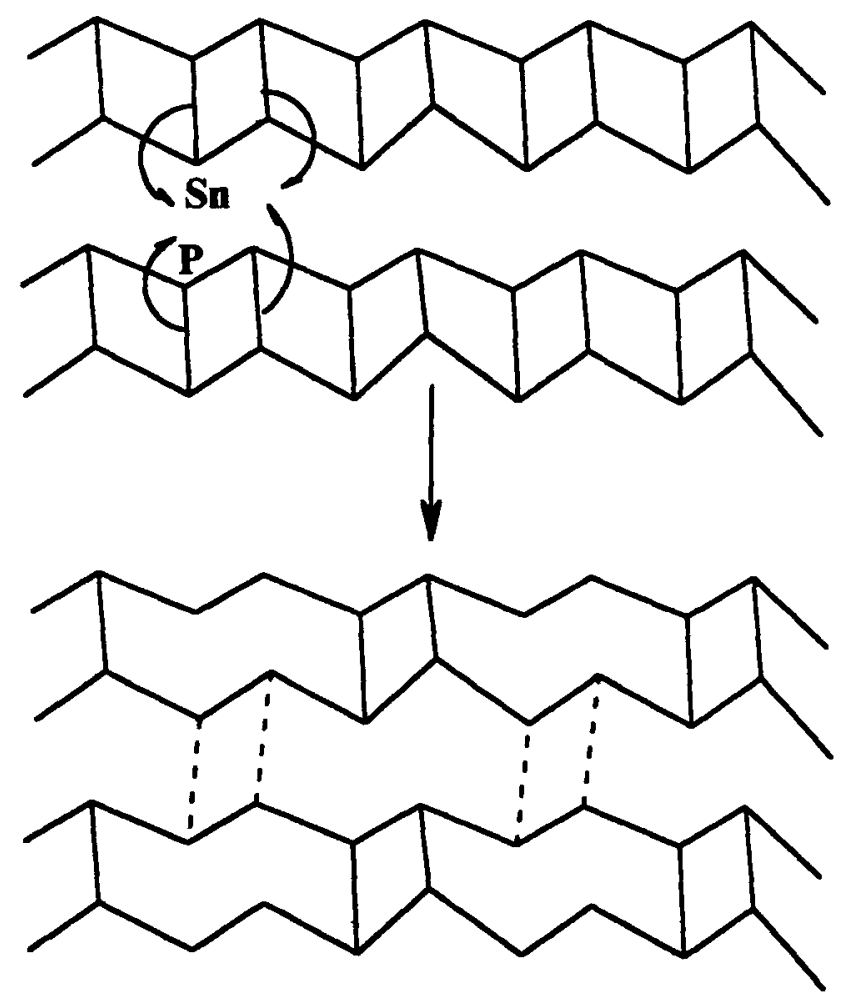

Figure 3. Conversion of the chain into the layered structure. See that 4-membered rings are broken to form 8-membered rings. Dotted lines represent the new 4- and 8-mantered rings formed due to $\mathrm{Sn}-\mathrm{O}-\mathrm{P}$ bonding and then the layer (from Ayyappan et al 1998a). six- and twelve-membered channels (figure 6a). The lone pair of electrons of the $\mathrm{Sn}^{11}$ protrude into the twelvemembered channel rendering it ineffective for sorption of a guest molecule. Along the $c$ axis, the 4-, 6- and 8-rings are connected to form a twelve-membered ring channel which has the appearance of a six-membered channel due to distortion. The amine and the water molecules are both present in this channel. Most of the distortions in the channels arise due to the three-coordination of the tin(II) with the oxygens in this structure. The Sn atoms coordinated to three oxygens occupy the vertex of a trigonal pyramid; the lone pair presumably occupies the fourth vertex of tetrahedron.

Since the synthesis of this $\mathrm{Sn}$ (II) phosphate, $\mathrm{V}$, involved the use of two different optical isomers of the branched organic diamine, we expected the isomers to be resolved and form different channels. We find, however, that the two amine isomers are located at the crystallographic centre of inversion in the structure creating a new type of disorder. A low-temperature single crystal X-ray diffraction study $(T=100 \mathrm{~K})$ showed no change in the disorder, indicating it to be static rather than dynamic.

Thermogravimetric analysis of $\mathbf{V}$ in an oxygen atmosphere showed a mass loss of ca. $3.9 \%$ at $125^{\circ} \mathrm{C}$ due to the removal of two molecules of water present in the channels (calc. $4.1 \%$ ). Careful experiments showed that the material (heated to $125^{\circ} \mathrm{C}$ ) reabsorbs the water on cooling and storing it for a few hours under atmospheric conditions (typically $4 \mathrm{~h}$ ). This feature is noteworthy as this is the first time such a complete reversible dehydration-hydration process has been observed in an openframework tin phosphate material. TGA studies also reveal that the protonated 1,3-diaminopentane is removed at $350^{\circ} \mathrm{C}$. The crystal structure collapsed after removal of the amine. Another important feature of this $\mathrm{Sn}$ (II)

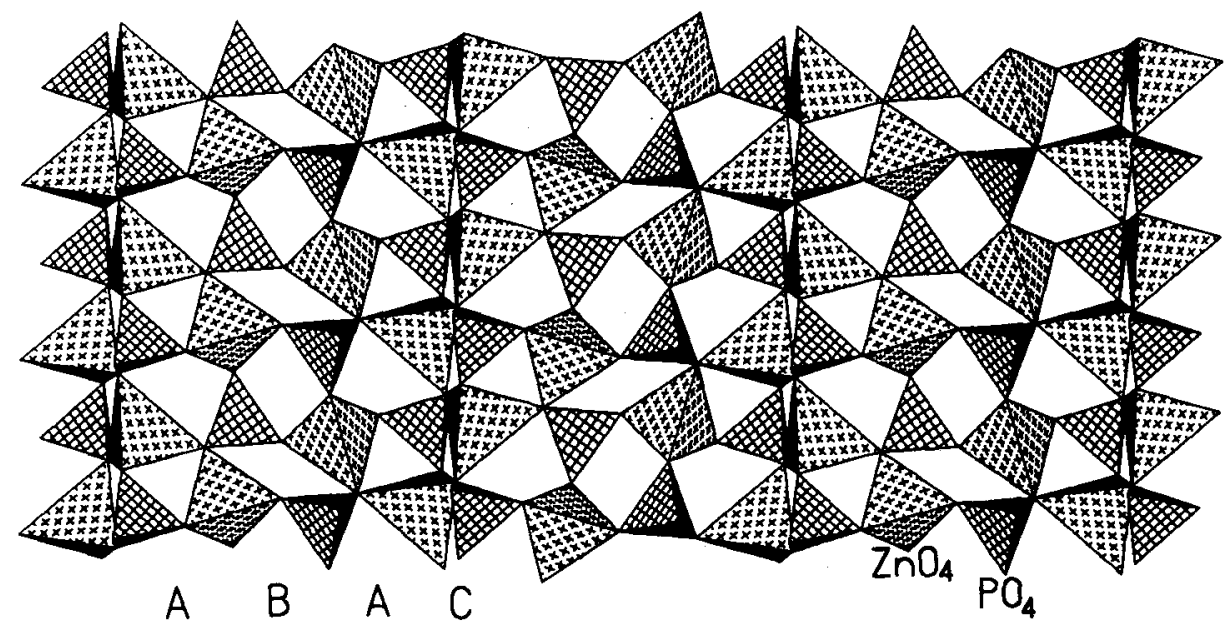

Figure 4. Polyhedral view of III, $\left[\mathrm{NH}_{3}\left(\mathrm{CH}_{2}\right)_{2} \mathrm{NH}\left(\mathrm{CH}_{2}\right)_{2} \mathrm{NH}_{3}\right]^{2+} 2\left[\mathrm{Zn}_{2} \mathrm{PO}_{4}\left(\mathrm{HPO}_{4}\right)\right]^{-}$along the $(010)$ direction showing the layers of various chains, and the connectivity between them. Note that the three-membered ring (C-type) forms the step in the layer (from Neeraj et al 1999a). 
phosphate is that the ammonium ions can be exchanged with $\mathrm{Na}^{+}$ions to a great extent.

Lest one should get the impression that only metal phosphates form open-framework structures, we will examine the structure of a Sn(II) oxalate (Ayyappan et al 1998c). The $\mathrm{Sn}(\mathrm{II})$ oxalate VI, has the composition $\left[\left(\mathrm{CH}_{3}\right)_{2} \mathrm{NH}\left(\mathrm{CH}_{2}\right)_{4} \mathrm{NH}\left(\mathrm{CH}_{3}\right)_{2}\right]^{2+}\left[\mathrm{Sn}_{2}\left(\mathrm{C}_{2} \mathrm{O}_{4}\right)_{3}\right]^{2-}$ with the individual layers consisting of a network of $\mathrm{SnO}_{6}$ and $\mathrm{C}_{2} \mathrm{O}_{4}$ moieties wherein each $\mathrm{Sn}$ atom is bound to six oxygens, in turn bound to carbon atoms forming the network. The oxalate ions are connected to the tin atoms forming the architecture. A remarkable feature of the structure of this material is the presence of 12 -membered rings within each inorganic sheet (figure 7). Such perforated sheets have been noticed earlier in a layered

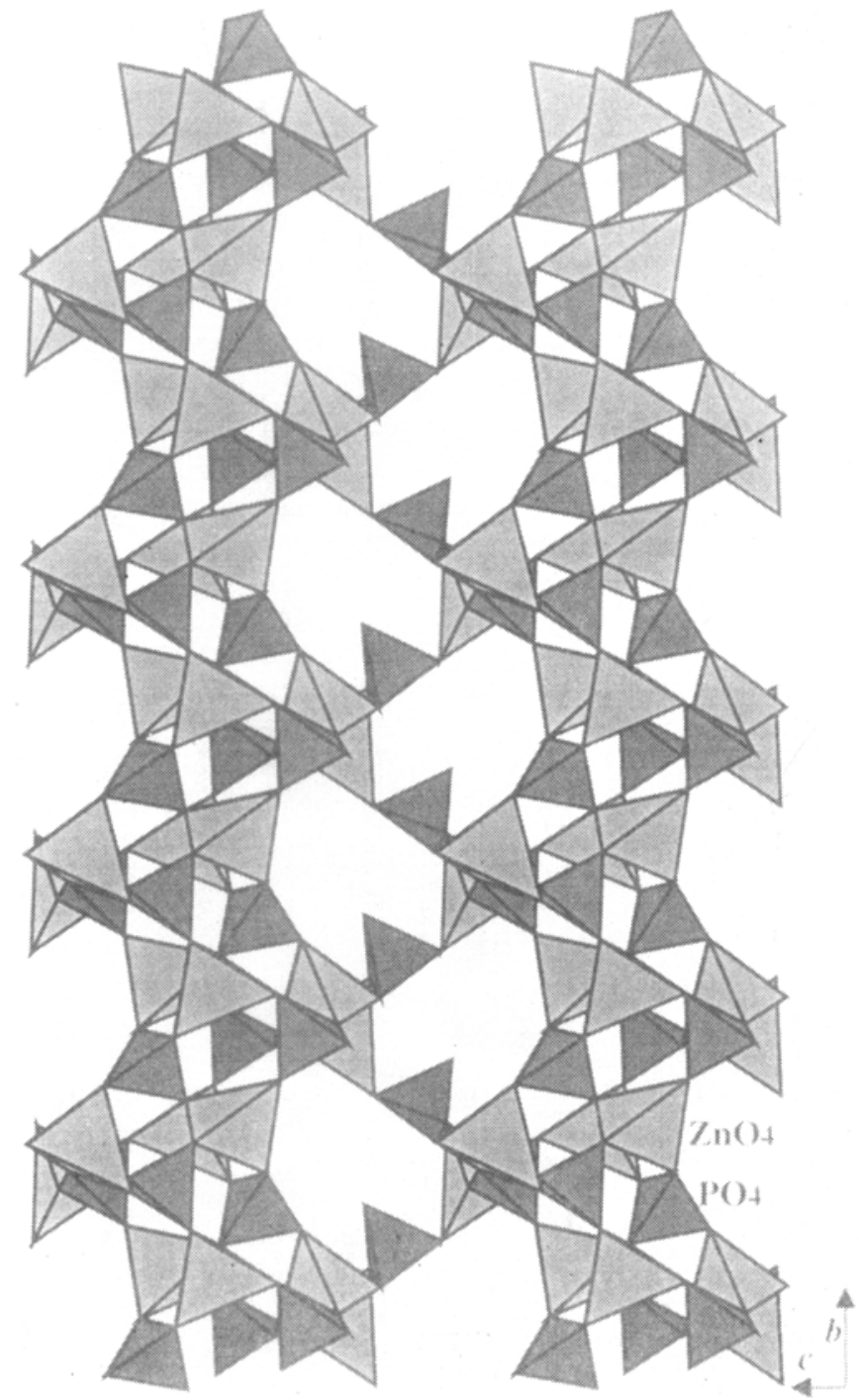

Figure 5. Structure of IV, $\left[\mathrm{NH}_{3}\left(\mathrm{CH}_{2}\right)_{2} \mathrm{NH}_{2}\left(\mathrm{CH}_{2}\right)_{2} \mathrm{NH}_{3}\right]^{3+}$ $\left[\mathrm{Zn}_{4}\left(\mathrm{PO}_{4}\right)_{3} \mathrm{HPO}_{4}\right]^{3-} \cdot \mathrm{H}_{2} \mathrm{O}$ showing the 8-membered channel (along the 100 direction) along with the helical channels (from Neeraj et al 1998b).
AIPO structure. The disposition of the molecules in the interlamellar organic layers is such that, in projection, the protonated $N, N, N^{\prime}, N^{\prime}$-tetramethyl-1,4-diaminobutane is in the middle of the pore formed by the 12-membered
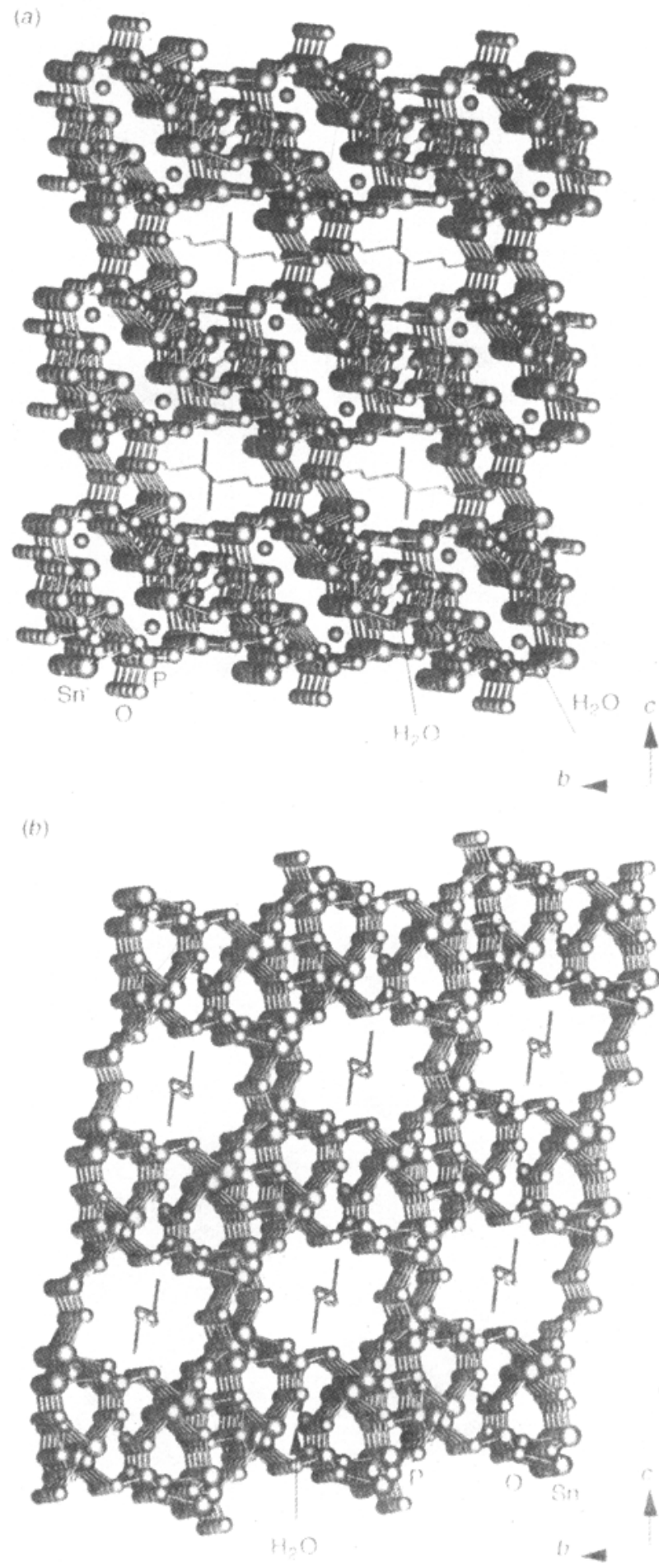

Figure 6. Structures of $\mathrm{V},\left[\mathrm{Sn}_{4} \mathrm{P}_{3} \mathrm{O}_{12}\right]^{-}\left[\mathrm{NH}_{3} \mathrm{CH}_{2} \mathrm{CH}_{2} \mathrm{CH}\left(\mathrm{NH}_{3}\right)\right.$ $\left.\mathrm{CH}_{2} \mathrm{CH}_{3}\right]_{0.5}^{2+} \cdot 2 \mathrm{H}_{2} \mathrm{O}$ (a) viewed down the $a$ axis and (b) the $b$ axis. Amine and water occupy different channels (from Natarajan et al 1998). 
rings. The pores penetrate the entire structure in a direction perpendicular to the sheets, thus yielding a solid with unidimensional channels $(9.486 \times 8.207 \AA$; longest atom-atom contact distance not including the van der Waals radii) similar to those in aluminosilicate zeolites such as Theta-1 and certain detemplated microporous AlPOs. The pores in the inorganic layers of VI result from the networking between six Sn atoms and six oxalate groups.

\section{Mesoporous solids}

Mesoporous solids have pores in excess of $20 \AA$, going upto $400 \AA$. In synthesizing these materials, one makes use of the self-organization of amphiphiles. The first synthesis of mesoporous solids was by Beck et al (1992) who prepared different forms of mesoporous silica. Many mesoporous oxide materials have since been prepared and characterized. We show the electron microscope image of a typical mesoporous sample in figure 8 . Since mesoporous solids are prepared by making use of selfassembled ordered aggregates of surfactants as templates, the structures exhibited by them are quite similar to those of the self-assembled surfactants. Accordingly, mesoporous solids occur in lamellar, hexagonal and cubic forms, just as the surfactant aggregates. Phase transitions among these forms occur in mesoporous materials in the solution phase. For example, changing the $\mathrm{pH}$ of the medium or ageing, leads to the transformation of the lamellar phase of silica to the hexagonal phase. Other factors such as temperature, presence of counter ions and concentration of the surfactant also affect the phase transitions. In surfactant systems, decreasing the concen-

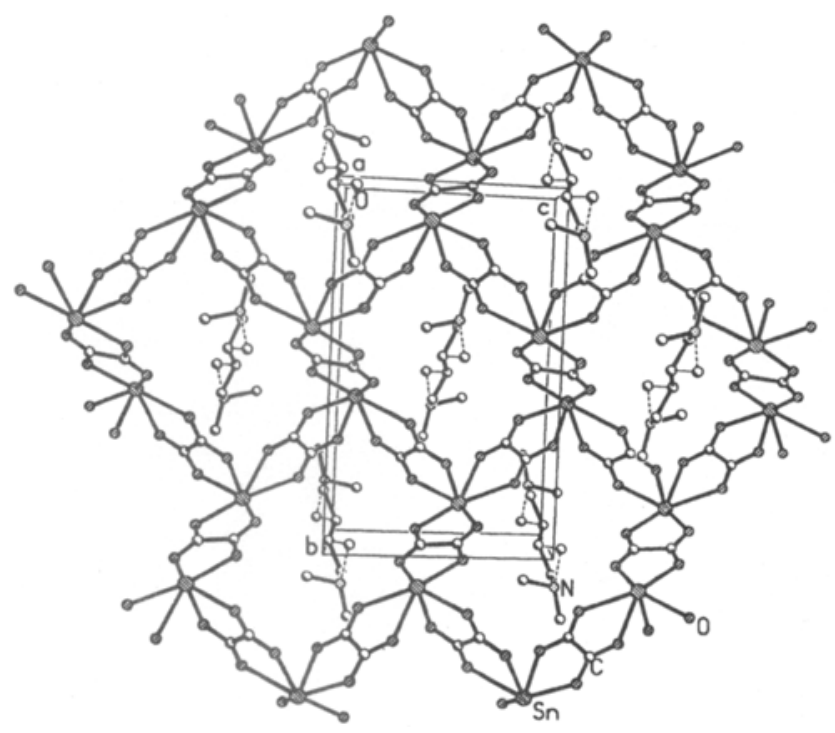

Figure 7. Structure of VI, $\left[\left(\mathrm{CH}_{3}\right)_{2} \mathrm{NH}\left(\mathrm{CH}_{2}\right)_{4} \mathrm{NH}\left(\mathrm{CH}_{3}\right)_{2}\right]^{2+}$ $\left[\mathrm{SN}_{2}\left(\mathrm{C}_{2} \mathrm{O}_{4}\right]_{3}\right]^{2-}$ viewed along the $a$ axis showing the 12-membered ring and the amine molecule (from Ayyappan et al 1998c). tration of the surfactant instantaneously transforms the lamellar phase to the hexagonal phase. The transitions in the surfactant self-assemblies are commonly understood in terms of the surface/interface energies of the ordered aggregates.

The effect of ageing on the lamellar to hexagonal phase transformation in mesoporous silica has been related to the extent of polymerization of the silica framework and the balancing of charges. High $\mathrm{pH}$ and a low degree of polymerization favour the lamellar phase whereas low $\mathrm{pH}$ and highly condensed silica favour the hexagonal phase of silica. The transformation from the hexagonal to the cubic phase in silica has been explained in terms of the formation of periodic minimal surface governed by a competition between the curvature and packing and the transformation is associated with kinetic barriers.

The kinetics of the lamellar $\rightarrow$ hexagonal $\rightarrow$ cubic transformations of mesoporous zirconia prepared by using a neutral organic amine as the amphiphile have been studied in phosphoric acid medium (Neeraj and Rao 1998). The lamellar $\rightarrow$ hexagonal transformation is preceded by a loss of the template molecules and the hexagonal $\rightarrow$ cubic transformation proceeds only when the lamellar form has entirely transformed to the hexagonal phase. The kinetics of the thermal transformation of lamellar zirconia to the hexagonal form have also been examined. This transformation is accompanied by a loss of template molecules as well. Accordingly, the activation energy of the lamellar-hexagonal transformation is comparable to the hydrogen bond energy

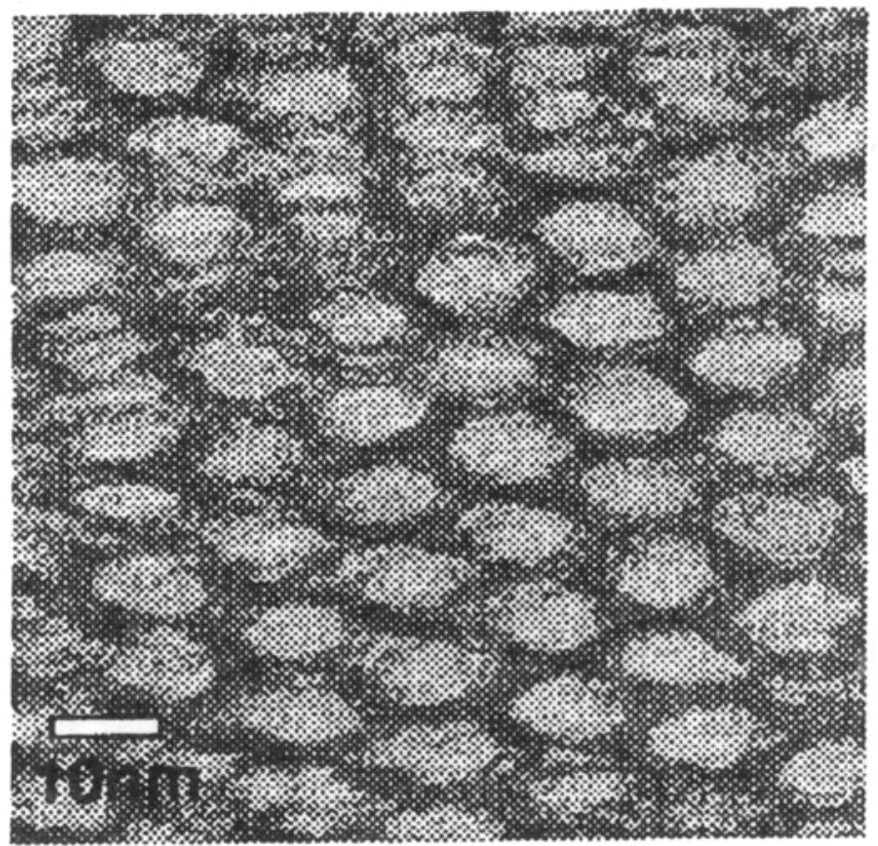

Figure 8. An electron microscope image of a mesoporous silica sample showing the ordered arrangement of large pores. 
between the amine and the oxo-zirconium species. The phase transformations of mesoporous zirconia can be understood in terms of minimum energy surfaces. In figure 9, we show the lamellar $\rightarrow$ hexagonal and hexagonal $\rightarrow$ cubic transformations schematically.

A study of the expansion of pore size in mesoporous silica by normal alkanes, $\mathrm{C}_{n} \mathrm{H}_{2 n+2}$, has revealed the important role of supramolecular organization making use of hydrophobic interactions (Ulagappan and Rao
1996). Hexagonal mesoporous silica prepared with hexadecyltrimethylammonium bromide shows a low angle reflection in the X-ray diffraction pattern at $39 \AA$ corresponding to $d_{1(0)}$. This suggests that the surfactant in the micelle is fully extended as in figure 10a. The $d_{100}$ increases progressively with the increase in the chain length of the alkane, reaching a value of $74 \AA$ for $n-\mathrm{C}_{14} \mathrm{H}_{30}$. A plot of $d_{100)}$ against the chain length of the alkane shows a linear increase from $n=9$ to 15 , coinciding
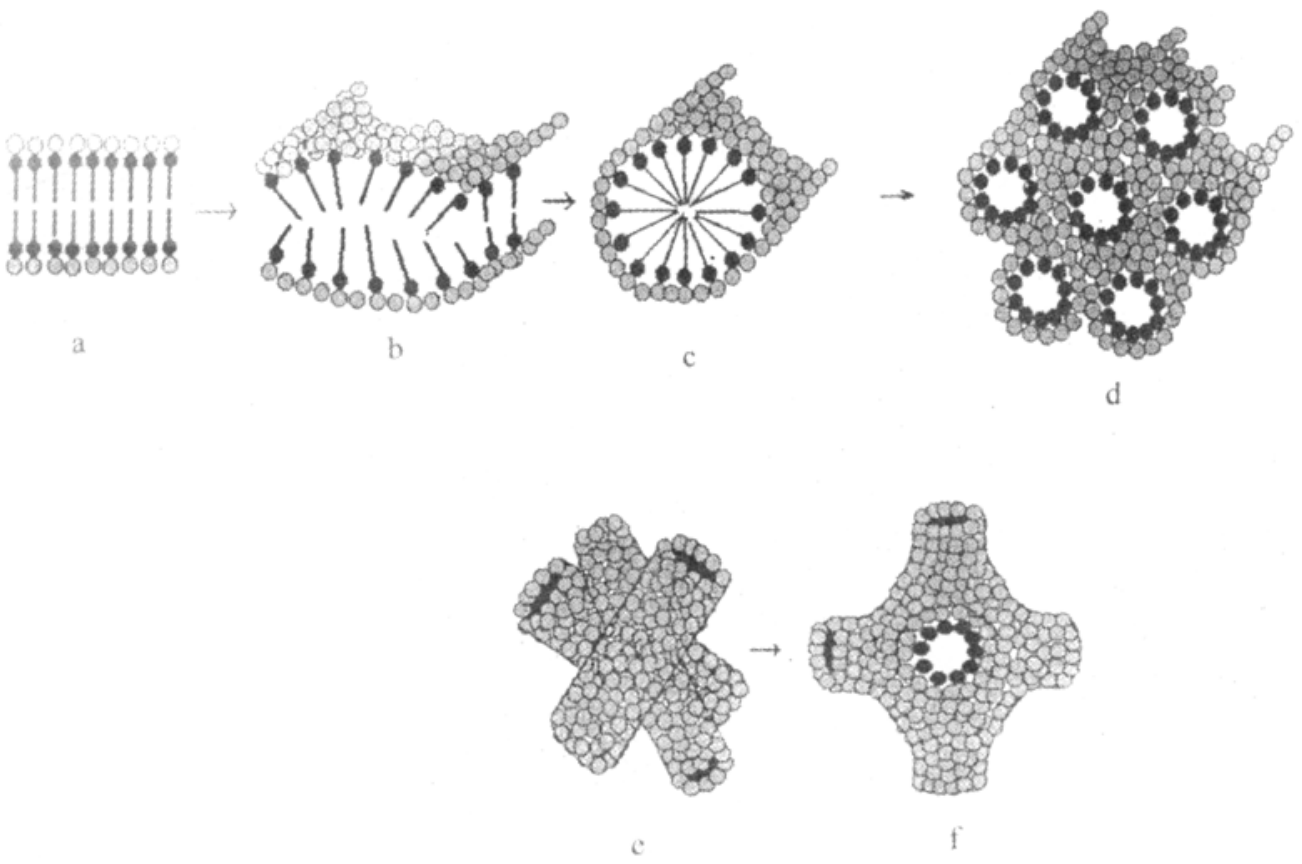

Figure 9. Schematic representation of the lamellar-hexagonal transformation (a to d) and hexagonal-cubic transformation (e and $\mathbf{n}$. The shaded circles around the surfactant aggregates represent the inorganic species, generally metal-oxo species (from Neeraj and Rao 1998).

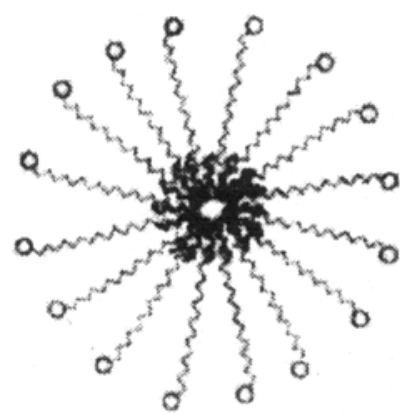

(b)

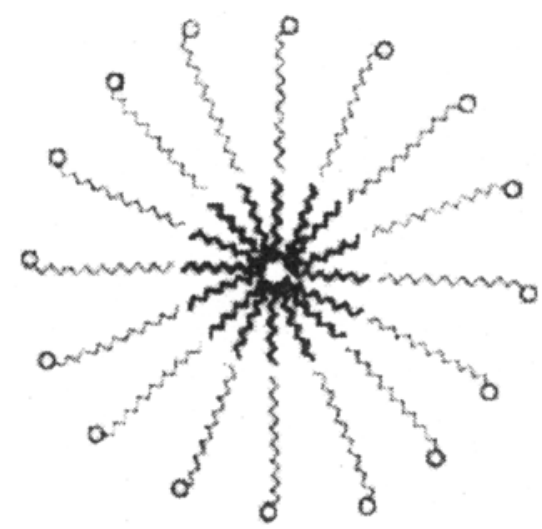

(c)

Figure 10. Schematic drawings of a micelle of surfactant molecules (a) in the absence of a solubilizing agent and (b), (c) in the presence of normal alkanes as solubilizing agents. The alkane molecules are represented by thicker lines (from Ulagappan and Rao 1996). 
with the theoretical line expected if there were additivity in the chain lengths of the alkane and the surfactant in forming the initial micelle. The $d_{100}$ values for alkanes with $n=5-8$ fall well below the theoretical line, suggesting that the entire chain length of the alkane is not involved in increasing the size of the micelle. Thus, hexane with a chain length of $6.4 \AA$ would be expected to increase the $d_{10 \%)}$ by about $12.8 \AA$ if the entire chain length were to contribute to the formation of the micelle, giving $d_{100}$ of $39+12.8=51.8 \AA$, whereas the observed $d_{10 m}$ value is $45.0 \AA$. The micelles formed with the $n=5-8$ alkanes and the surfactant molecules can be described as molecular dispersions of the solubilizing agent between the tails of the surfactant molecules as depicted in figure 10b. With the higher alkanes $(n=9-15)$, on the other hand, the increase in $d_{100}$ values is close to the theoretical values. For example, with $\mathrm{C}_{14} \mathrm{H}_{30}$ (chain length of $16.6 \AA$ ), the expected increase
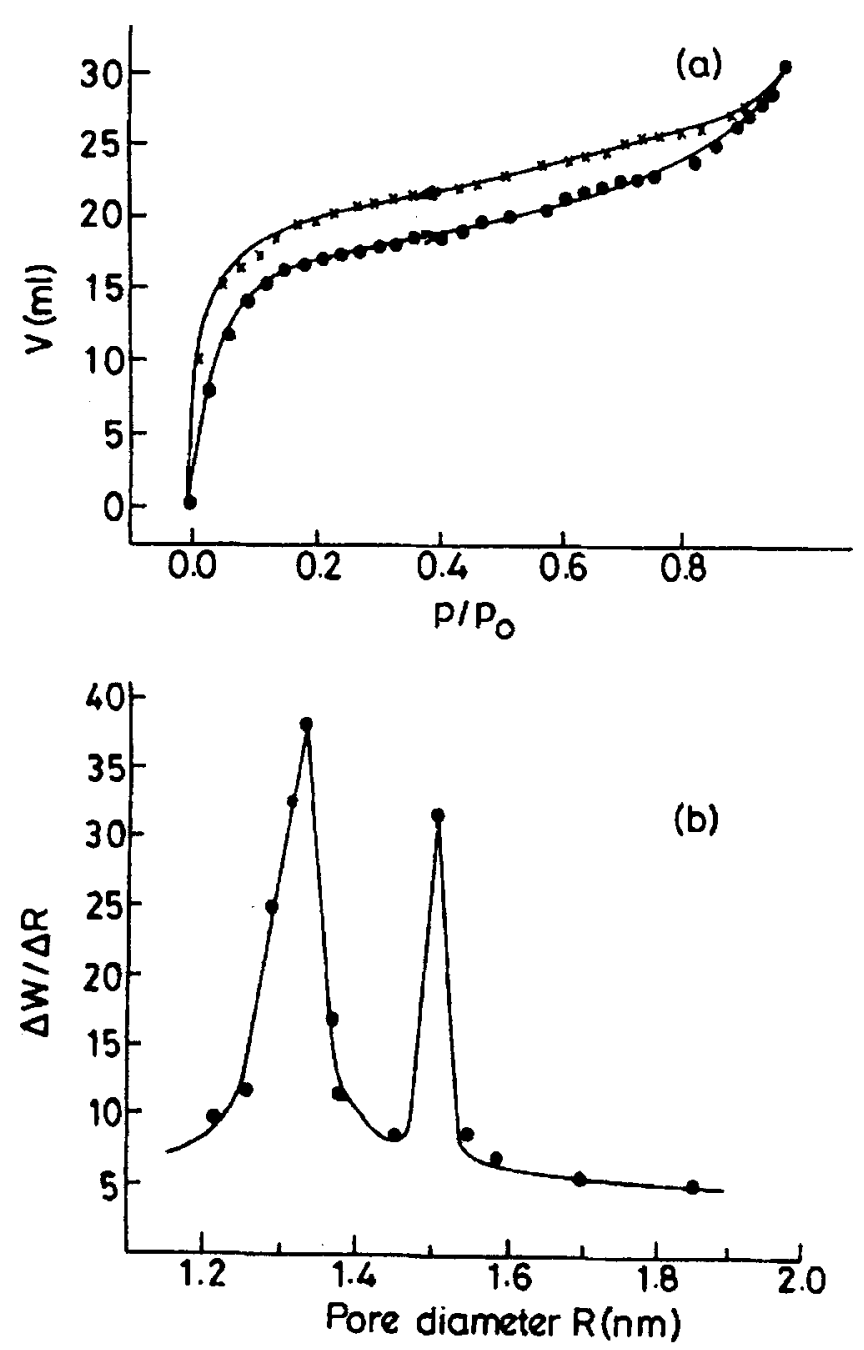

Figure 11. (a) $\mathrm{N}_{2}$ adsorption isotherm $(77 \mathrm{~K})$ and (b) pore-size distribution in microporous silica prepared by supramolecular templating of $n-\mathrm{C}_{6} \mathrm{H}_{13} \mathrm{NH}_{2}$ (from Easwaramoorthy et al 1999). in $d_{100}$ would be $33.2 \AA$, predicting a $d_{100}$ value of $39+33 \cdot 2=72.2 \AA$; the observed $d_{100}$ of $73.6 \AA$ is quite close to this value. Alkanes with $n>15$ do not show such additivity (e.g. $d_{100}$ with $n=16$ is $66.9 \AA$ ) suggesting that the chains may not be rigid.

The behaviour of the $n=9-15$ alkanes is best described by figure 10c where the alkane molecules form a core which is then surrounded by a layer of the cationic surfactant molecules, with a one-to-one alignment of the alkane chain and the surfactant tail. This is an extraordinary example of supr amolecular organization based on hydrophobic interaction. Such a cooperative organization ar ising from hydrophobic interaction lends credence to the expectation that the structure-directing effect of organic molecules may make it possible to design inorganic solids.

It was mentioned earlier that mesoporous solids have pore diameters larger than $2 \mathrm{~nm}$. Microporous solids generally have pore diameters between 0.5 and $1 \mathrm{~nm}$, although the general range given is $0.5-2 \mathrm{~nm}$. There are very few microporous solids, however, with the intermediate range pore diameters of 1-2 nm. Recently, hexagonal microporous phases of $\mathrm{SiO}_{2}$ and $\mathrm{AlPO}_{4}$ with pore sizes in the intermediate range have been prepared by making use of the supramolecular templating of the short-chain amine, $n-\mathrm{C}_{6} \mathrm{H}_{13} \mathrm{NH}_{2}$ (Easwaramoorthy et al 1999). Pore diameters of $\sim 1.4 \mathrm{~nm}$ have been obtained by this means in both the cases. The surface area of $\mathrm{SiO}_{2}$ after removal of the template was $800 \mathrm{~m}^{2} \mathrm{~g}^{-1}$. In figure 11, we show the $\mathrm{N}_{2}$ adsorption isotherm (type $\mathrm{I}$ similar to that in zeolites) and the pore size distribution in microporous silica prepared in the manner described above.

\section{Organic porous solids}

In the last few years, there has been some effort to design organic porous solids by supramolecular organization. Channel structures formed by trimesic acid are an example. We give here two outstanding examples of organic channel structures obtained recently in this laboratory. First, we examine the structure of the adduct obtained by the cocrystallization of trithiocyanuric acid (TTCA) with 4,4'-bipyridine (BP) from a methanolbenzene solution (Pedireddi et al 1997). These crystals had TTCA, BP and benzene in the molar ratio of $2: 1: 1$. TTCA and BP form a hydrogen bonded network with strong $\mathrm{N}-\mathrm{H} \ldots \mathrm{N}$ and $\mathrm{N}-\mathrm{H} \ldots \mathrm{S}$ hydrogen bonds (as well as weak $\mathrm{C}-\mathrm{H} \ldots \mathrm{S}$ bonds), and the network has a large cavity (figure 12a). The three-dimensional structure has channels where the benzene molecules reside as shown in figure $12 \mathrm{~b}$. Benzene is removed on heating but can be reabsorbed. The channel is also shape-selective, in that it accommodates para-xylene, but not the ortho and meta isomers. 
An even more impressive example of a channel structure is that formed by the $1: 1$ hydrogen bonded adduct of cyanuric acid (CA) and melamine (M). That, the adduct between these two molecules should give a beautiful rosette structure had been predicted by Whitesides some years ago. It has at last been possible to prepare this adduct by hydrothermal means (Ranganathan et al 1999).
In figure 13a we show the rosette formed in the adduct between $\mathrm{CA}$ and $\mathrm{M}$, with a cavity of $\sim 4 \AA$. The three-dimensional arrangement of the adduct with channels is shown in figure $13 \mathrm{~b}$. In this structure, every possible bonding opportunity has been exploited by the molecules. In the plane of the adduct in figure $11 \mathrm{a}$, every $\mathrm{CA}$ or $\mathrm{M}$ molecule forms six hydrogen bonds

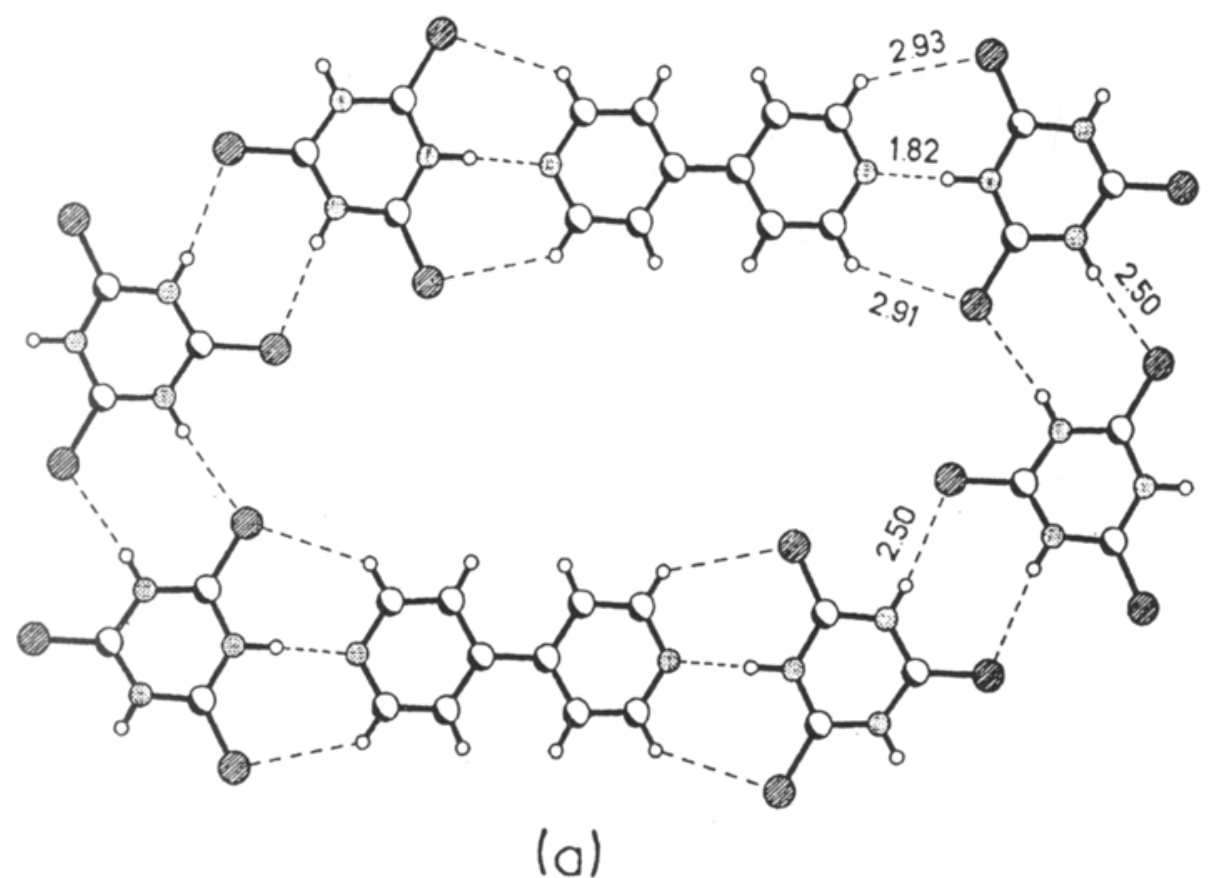

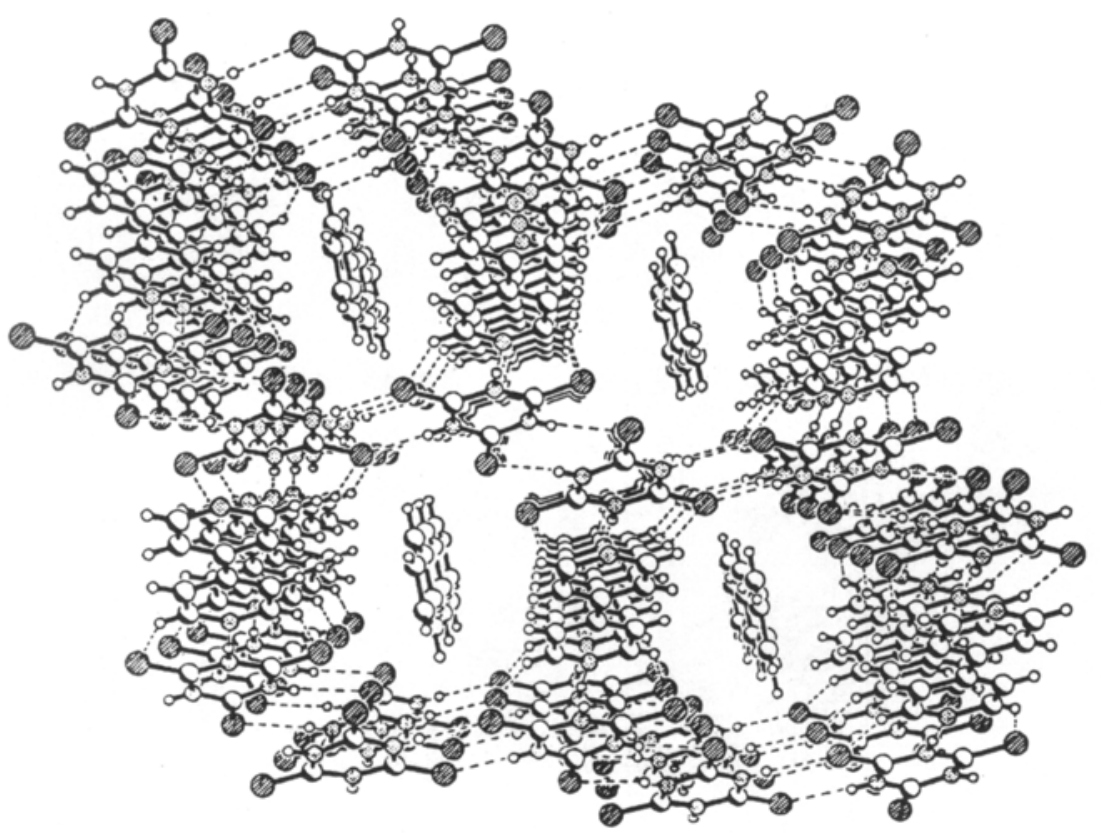

(b)

Figure 12. (a) Self-assembly of TTCA and BP molecules forming a layered network with large cavities and (b) channels in the three-dimensional arrangement, occupied by benzene molecules (from Pedireddi et al 1997). 

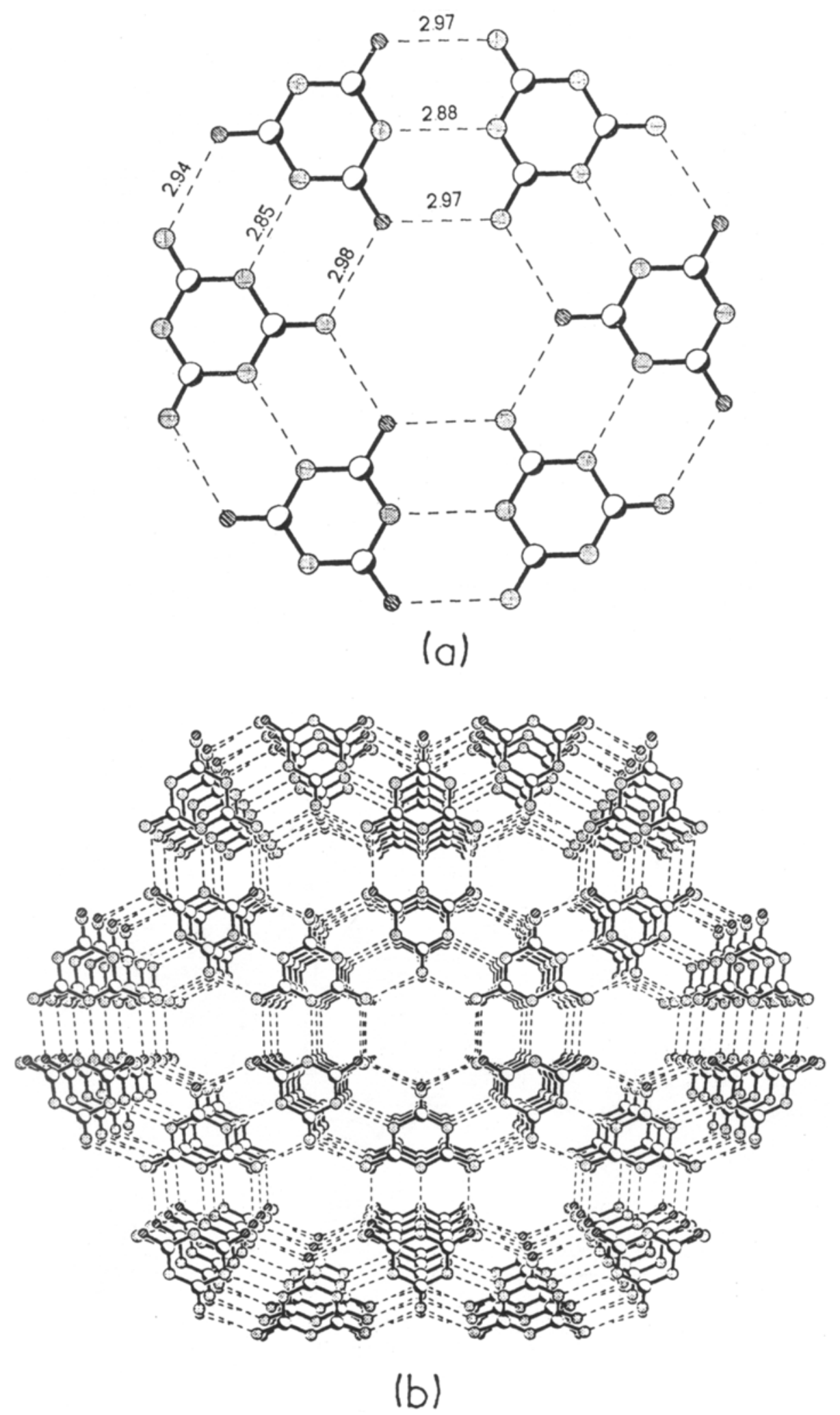

Figure 13. (a) $A$ rosette formed in adduct of $C A$ and $M$ molecules with a cavity diameter of $4 \AA$. Dashed lines represent hydrogen bonds. (b) Three-dimensional arrangement of the CAM adduct showing channels along the $c$ :axis (from Ranganathan et al 1999). 
(three with the neighbour on either side). Further bonding between molecules occurs by the stacking of the rosettes in such a way that $\mathrm{CA}$ and $\mathrm{M}$ molecules alternate in the direction perpendicular to the rosette.

\section{Acknowledgement}

The author thanks his coworkers, in particular Prof. A K Cheetham, Dr S Natarajan, Dr S Ayyappan, Dr M Easwaramoorthy, Dr V R Pedireddi and Mr S Neeraj, for most fruitful collaboration in some of the studies discussed in the article.

\section{References}

Atwood J L, Davies J E D, Mac Nicol D D and Vogtle F (eds) 1996 Comprehensive supramolecular chemistry (Oxford: Pergamon)

Ayyappan S, Bu X, Cheetham A K, Natarajan S and Rao C N R 1998a Chem. Commun. 2181
Ayyappan S, Bu X, Cheetham A K and Rao C N R 1998b Chem. Mater. 103308

Ayyappan S, Cheetham A K, Natarajan S and Rao C N R 1998c Chem. Mater. 103746

Beck J S et al 1992 J. Am. Chem. Soc. 11410834

Bowes C L and Ozin G A 1996 Adv. Mater. 813

Easwaramoorthy M, Neeraj S and Rao C N R 1999 Microporous Mesoporous Mater. 28205

Natarajan S, Easwaramoorthy M, Cheetham A K and Rao C N R 1998 Chem. Commun. 1561

Neeraj S and Rao C N R 1998 J. Mater. Chem. 81631

Neeraj S, Natarajan S and Rao C N R 1999a Chem. Mater. 11

Neeraj S, Natarajan S and Rao C N R 1999b Chem. Commun. 165

Pedireddi V R, Chatterjee S, Ranganathan A and Rao C N R 1997 J. Am. Chem. Soc. 11910867

Ranganathan A, Pedireddi V R and Rao C N R $1999 \mathrm{~J}$. Am. Chem. Soc. 1211752

Rao C N R 1999 J. Mater. Chem. 91

Thomas J M 1994 Angew Chem. Intl. Ed. Engl. 33913

Ulagappan N and Rao C N R 1996 Chem. Commun. 2759 